\title{
MEDIDAS ALTERNATIVAS DE VOLATILIDAD EN EL MERCADO DE VALORES PERUANO
}

\section{RESUMEN}

Este documento busca comparar las principales metodologías de cálculo de la volatilidad para el mercado de valores peruano. Se presentan tres métodos de cálculo de volatilidad, el modelo EWMA, el modelo GARCH y el de Volatilidad estocástica(SV). La comparación de estas metodologías se realizó a través del cálculo del Valor en Riesgo y un ejercicio de backtesting. Los resultados muestran que si bien las tres metodologías de estimación generan medidas de volatilidad similares, los modelos GARCH y SV son superiores al modelo EWMA en términos del cálculo del Valor en Riesgo. Asimismo, el ejercicio de backtesting realizado no muestra diferencias significativas entre los modelos GARCH y de volatilidad estocástica.

JEL: G10, G11, G12.

PALABRAS CLAVES: Volatilidad estocástica, valor en riesgo, y estrategia de trading.

\begin{abstract}
This paper aims to compare the main methodologies to estimate the volatility of Peruvian stock market. Three main methods for volatility estimation are introduced, EWMA model, GARCH model and Stochastic Volatility (SV) model. To compare these methods we use a Value at Risk application and a backtesting exercise. The results shows that all three methods provide similar volatility estimates, however GARCH and SV models are superior to EWMA model in terms of Value at Risk estimates. Moreover, the backtesting exercise implemented does not show significant differences between the GARCH model and the SV model.
\end{abstract}

JEL: G10, G11, G12.

KEYWORDS: Stochastic Volatility, Value at Risk, and Backtesting.

\footnotetext{
A Investigador Docente de la Escuela Profesional de Economía. Facultad de Ciencias Contables, Económicas y Financieras de la Universidad de San Martín de Porres. Se agradece enormemente la asistencia de investigación de Ángel Torres y Luis Soriano quienes han realizado un excelente trabajo. Las opiniones vertidas en este documento no necesariamente coinciden con las de la USMP ni con las de otras instituciones a las que pertenece el autor.
} 


\section{INTRODUCCIÓN}

Una de las características propias a los retornos financieros (basados en activos financieros como las acciones, índices, tipos de cambio, entre otros) es que la volatilidad no es constante en el tiempo (existen clusters de volatilidad). Por tal motivo, los retornos financieros usualmente se estiman considerando modelos que permiten que la volatilidad cambie en el tiempo. Generalmente, esta volatilidad cambiante en los retornos financieros se modela utilizando modelos tipo EWMA (Riskmetrics) o GARCH, en los cuales la evolución de la volatilidad se modela de forma determinística (Engle, 1982; Bollerslev, 1986; y todas las variantes posteriores).

Sin embargo, alternativamente se puede modelar dicho comportamiento de los retornos financieros utilizando modelos probabilísticos de volatilidad - considerando Modelos de Volatilidad Estocástica (Taylor, 1982; Kim, Shephard y Shib, 1998; entre otros). Si bien estos autores dan argumentos sólidos para preferir los Modelos de Volatilidad Estocástica (SV), su uso práctico ha tenido menor interés en comparación al uso extensivo de los modelos GARCH. Al respecto, Bos (2012) señala que esta inconsistencia se debe a que los modelos SV carecen de una metodología única de estimación, así como la ausencia de un único software que concentre los distintos métodos de estimación.

Como respuesta, Kastner y Fruhwirth-Schnatter (2014) propone un método de estimación eficiente utilizando Markov Chain Montecarlo (MCMC). Esta nueva metodología de estimación de los modelos de volatilidad estocástica ha permitido extender el uso de los modelos SV en los casos que se requiera cuantificar la volatilidad de los retornos financieros.

En ese sentido, el presente artículo busca aplicar esta nueva metodología de estimación al retorno del mercado bursátil peruano y determinar sus ventajas con respecto a los modelos tradicionales.

\section{REVISIÓN DE LA LITERATURA}

Existe consenso en la literatura de precio de activos financieros en relación a las características comunes que poseen los distintos retornos financieros, tales como tipo de cambio e índices bursátiles. Dichos retornos presentan a través del tiempo periodos estables y periodos de elevada incertidumbre (clusters), los cuales reflejan la situación económica doméstica e internacional de un país o región, lo que hace importante modelar la varianza de dichos retornos para analizar su dinámica a través del tiempo.

Inicialmente, esta volatilidad cambiante se modeló a través del modelo EWMA o Riskmetrics (JP Morgan, 1994) dada su facilidad para implementar. Sin embargo, en la historia más reciente dos metodologías alternativas se han desarrollado para lograr esta volatilidad cambiante: en primer lugar, se destacan los modelos cuya volatilidad tiene una modelación determinística (conocidos como modelos tipo GARCH), y en segundo lugar, los modelos que presentan volatilidad modelada de forma estocástica (Stochastic Volatility Models, $\mathrm{SV}$ ). Los modelos GARCH suponen que la volatilidad (varianza) evoluciona en el tiempo siguiendo un proceso determinado por la dependencia determinística entre el retorno del activo financiero y su varianza, mientras que los modelos SV son más flexibles y consideran que la varianza sigue un proceso que puede fluctuar de manera independiente a los retornos del activo financiero. Si bien a través de los años se hizo extensivo el uso de los modelos tipo GARCH para el cálculo de la volatilidad de los retornos financieros, la evidencia empírica sobre las bondades de los modelos de volatilidades estocásticas ha sido escasa y estudiada más recientemente.

Alanya y Rodríguez (2014) compararan la eficiencia de tres algoritmos para la estimación de Modelos SV y dos modelos tipo GARCH (N-GARCH y t-GARCH).El primer método es el MCMC (cadena de Markov Monte Carlo) usando el algoritmo de la Gibbs-sampling, asumiendo un comportamiento de distribución normal estándar del error para la estimación de los coeficientes del modelo. Esta metodología se centra en calcular la distribución posterior del conjunto de parámetros a partir de la distribución de los parámetros anteriores mediante simulaciones de Montecarlo. El segundo método es llamado combinación de muestras: a partir de la linealización del modelo, el término de error es aproximado mediante una combinación de distribuciones normales. Finalmente, el tercer método es la integración de muestras, que consiste en integrar o separar las volatilidades del proceso muestral con el objetivo de obtener una mejora en la aleatoriedad y disminuir la correlación que existe entre los parámetros y la varianza.

Usando datos de los retornos de activos financieros como el índice bursátil (Índice 
General de la Bolsa de Valores de Lima) y el retorno cambiario (variación del tipo de cambio) al cierre del día desde diciembre 1992 hasta diciembre del 2010, Alanya y Rodríguez (2014) encuentran que el método de muestras integradas es el más eficiente. Por otro lado el método de Gibbs-sampling proporciona grandes valores de volatilidad sobre todo en los períodos de alta incertidumbre en relación a los otros dos métodos.

Por su parte, Lengua Lafosse y Rodríguez (2018) presenta un análisis empírico los Modelos de Volatilidad Estocástica (SV) de los retornos de los mercados de renta variable de un conjunto de países de Latinoamérica (Perú', Argentina, Chile, Brasil y México) considerando una muestra que comprende el período entre 1996 y 2013. Los autores encuentran que hay efectos de apalancamiento en todos los 'índices bursátiles considerados, pero no hay suficiente evidencia para el Perú', y perturbaciones sesgadas con colas pesadas es confirmado sólo para Argentina, cola pesada simétricas para México, Brasil y Chile, y las normalidad simétrica para el Perú'. Semejante a los hallazgos de Nakajima, J. y Omori, Y. (2009).

Adicionalmente, Lafosse y Rodriguez (2018) encuentran que la media posterior de los parámetros estimados es consistentes con la literatura que indican la alta persistencia de la volatilidad de los retornos del mercado de valores. Sin embargo, en base a los cálculos obtenidos se muestra que los rendimientos IGBVL tienen una menor persistencia en comparación con la volatilidad de los índices de los demás índices bursátiles de América Latina considerados. Los resultados apoyan la evidencia de que existen efectos de apalancamiento en todos los índices considerados, pero no hay suficiente evidencia para la IGBVL. Otro resultado importante es que la volatilidad (en logaritmos) de los rendimientos IGBVL tiene más variabilidad que los otros rendimientos de las acciones en América Latina. Además, los resultados apoyan la evidencia de colas pesadas y sesgadas sólo para el MERVAL, colas pesada simétricas para el Mexbol, IBOVESPA e IPSA, y normalidad el IGBVL.

\section{MODELOS DE VOLATILIDAD}

Para caracterizar la volatilidad del índice bursátil peruano, se utilizarán tres modelos de varianza cambiante en el tiempo: EWMA, GARCH y volatilidad estocástica. A continuación se describe las principales características de cada uno de estos modelos.

\section{a. Modelo Exponentially Weighted Moving Average (EWMA o Riskmetrics)}

Este modelo es una extensión simple de promedio ponderado tradicional de todas las observaciones, siendo las observaciones más recientes las que reciben mayor ponderación.

$$
\sigma_{t}^{2}=(1-\lambda) r_{t-1}^{2}+\lambda \sigma_{t-1}^{2}
$$

Es decir, es un promedio móvil ponderado exponencialmente, donde las últimas observaciones tienen mayor ponderación:

$$
\sigma_{t}^{2}=\sum_{j=1}^{\infty}(1-\lambda) \lambda^{j-1} r_{t-j}^{2}, \quad 0<\lambda<1
$$

Con un valor de la varianza al inicio de la muestra $\mathrm{y}$ a partir de un valor de $\lambda$ se puede obtener un estimador de la volatilidad que cambia en el tiempo.

\section{MODELO GARCH}

Bollerslev (1986) propuso un ARCH generalizado $(\mathrm{GARCH})$ de orden $(1,1)$ :

$$
\sigma^{2}=\alpha_{0}+\alpha_{1} r^{2}+\beta_{1} \sigma^{2}
$$

A diferencia del modelo EWMA, el modelo GARCH requiere la estimación de los parámetros de la ecuación de la varianza, y así obtener un estimado de la volatilidad.

\section{b. Modelo de Volatilidad Estocástica (SV)}

Siguiendo a Kastner (2016), denotamos por rt = $(\mathrm{r} 1, \mathrm{r} 2, \ldots, \mathrm{rt})$ ' a un vector de retornos financieros con media zero. La característica particular de los modelos de volatilidad estocástica (SV) es que cada observación rt se asume que tiene su propia varianza contemporánea eht, relajando así el supuesto de homocedasticidad. Para que la estimación de un modelo de este tipo sea posible, no se permite que la varianza varíe de manera irrestricta con el tiempo. Más bien, se asume que su logaritmo sigue un proceso autorregresivo de orden 1. Es importante tomar en cuenta que esta característica tiene diferencias fundamentales con respecto a los modelos tipo GARCH, donde la volatilidad variante en el tiempo se asume que sigue una dinámica determinística en lugar de una dinámica estocástica como en los procesos SV. 
Un modelo de volatilidad estocástica (SV) puede expresarse de manera conveniente de una forma jerárquica. Usualmente se expresan de la siguiente manera:

$$
\begin{aligned}
r_{t} \mid V_{t} & \sim \mathrm{N}\left(\mathrm{o}, e^{V t}\right) \\
V_{t} \mid V_{t-1}, \mu, \phi, \sigma_{\eta} & \sim \mathrm{N}\left(\mu+\theta\left(V_{t-1}-\mu_{\eta}\right), \sigma^{2}\right) \\
V_{0} \mid \mu, \phi, \sigma_{\eta} & \sim \mathrm{N}\left(\mu, \sigma_{\eta}^{2}\right) /\left(1-\theta^{2}\right)
\end{aligned}
$$

donde $\left.N\left(\mu, \sigma^{2}\right)\right)_{\eta}$ denota la distribución normal con media $\mu$ y varianza ). Asimismo, denotaremos $\left.\Omega=\left(\mu, \varphi, \sigma_{\eta}^{2}\right)\right)^{\prime}$ como el vector de parámetros: $\mu$ el nivel del logaritmo de la varianza; la persistencia del logaritmo de la varianza: y ơ la volatilidad del logaritmo de la varianza. El proceso $\mathbf{V}_{t}=\left(V_{0}, V_{1}, \ldots, V_{n}\right)$ ' que parece en la segunda y tercera ecuación es un proceso no observado y se interpreta como la volatilidad cambiante en el tiempo (de manera más precisa del logaritmo de la volatilidad). Finalmente, $V_{0}$ que aparece en la ecuación 3 se distribuye de acuerdo a la distribución estacionaria de proceso autorregresivo de orden 1.

\section{DATOS Y RESULTADOS}

Para implementar el cálculo de volatilidad en el mercado de valores peruano se utiliza los datos diarios del retorno de la Bolsa de Valores de Lima (Índice general, IGBVL) para una muestra que comprende el período entre enero de 2010 y diciembre de Mayo de 2019. Este horizonte de análisis permite analizar el periodo post-crisis financiera y caracterizar como la volatilidad de los retornos bursátiles de la BVL ha evolucionado durante dicho periodo.

Para calcular la volatilidad a partir del modelo EWMA, el proceso de cálculo iterativo empieza con un valor inicial de la varianza igual a la varianza de las primeras 30 observaciones y los siguientes valores se calculan a partir del modelo descrito en la sección 3.1 considerando que $\lambda=0.94$.

A partir del cálculo de la volatilidad utilizando la metodología de EWMA se puede identificar algunos períodos con elevada volatilidad. Por ejemplo, en el periodo 2011-2012 asociado a la crisis europea, e periodo del "taper tantrum" en el año 2013 y finalmente el periodo 2015-2016 asociado a los riesgos de desaceleración china y caída en los precios de commodities (Gráfica 1).

\section{Gráfica 1. Medición de volatilidad del IGBVL utilizando la metodología EWMA}

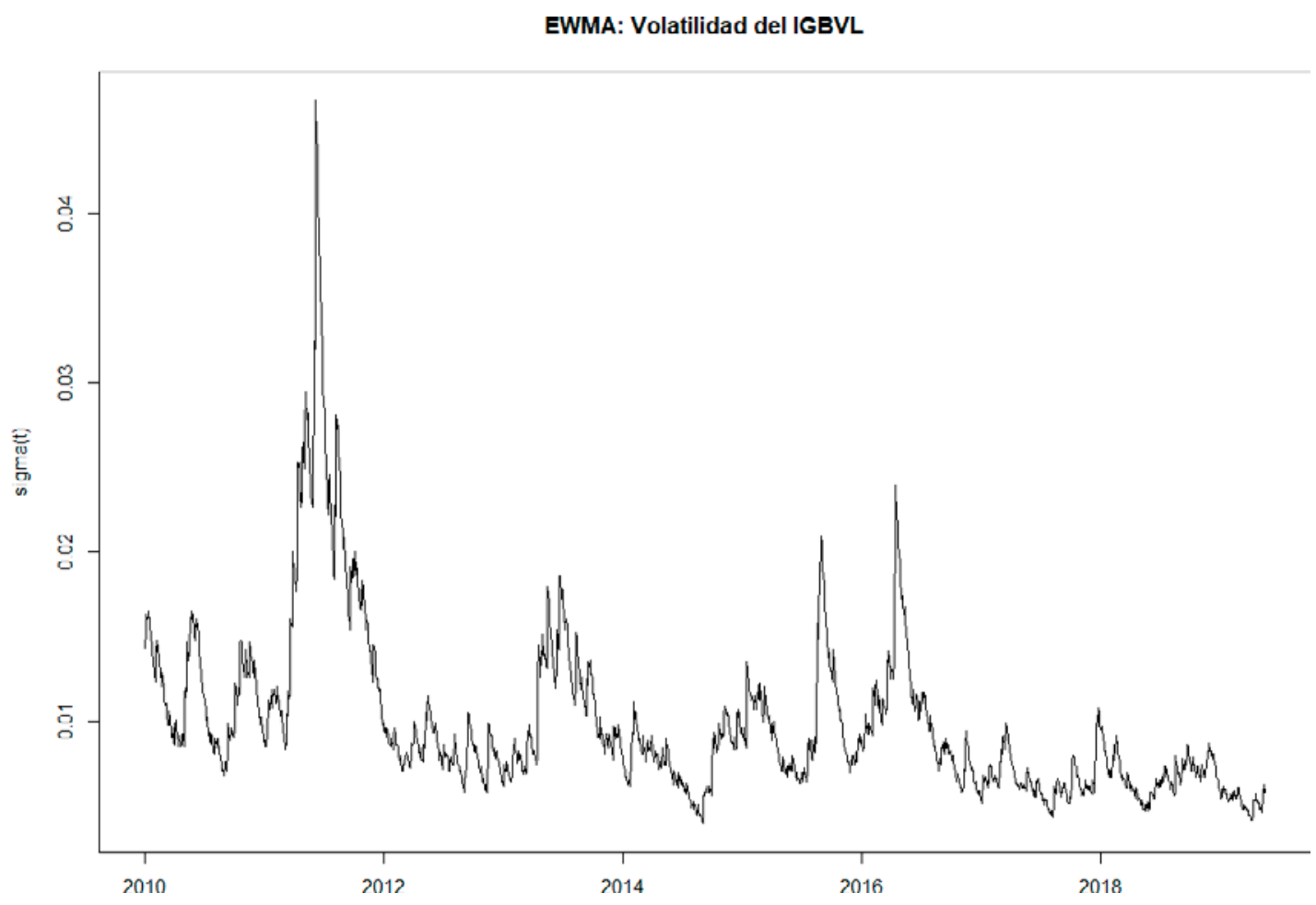


Sin embargo, como se indicó anteriormente, en el modelo EWMA los coeficientes que se utilizan para el cálculo de la volatilidad no son estimados sino se utiliza un valor fijo. Una aproximación donde se puede considerar que sean los datos los que determinen los coeficientes requeridos para el cálculo de la volatilidad se realiza a partir de un modelo GARCH. La especificación utilizada para la volatilidad es un GARCH $(1,1)$ con residuos estandarizados que se asumen siguen una distribución normal (Gráfica 2).

\section{Gráfica 2. Medición de volatilidad del IGBVL utilizando la metodologia GARCH}

GARCH: Volatilidad del IGBVL

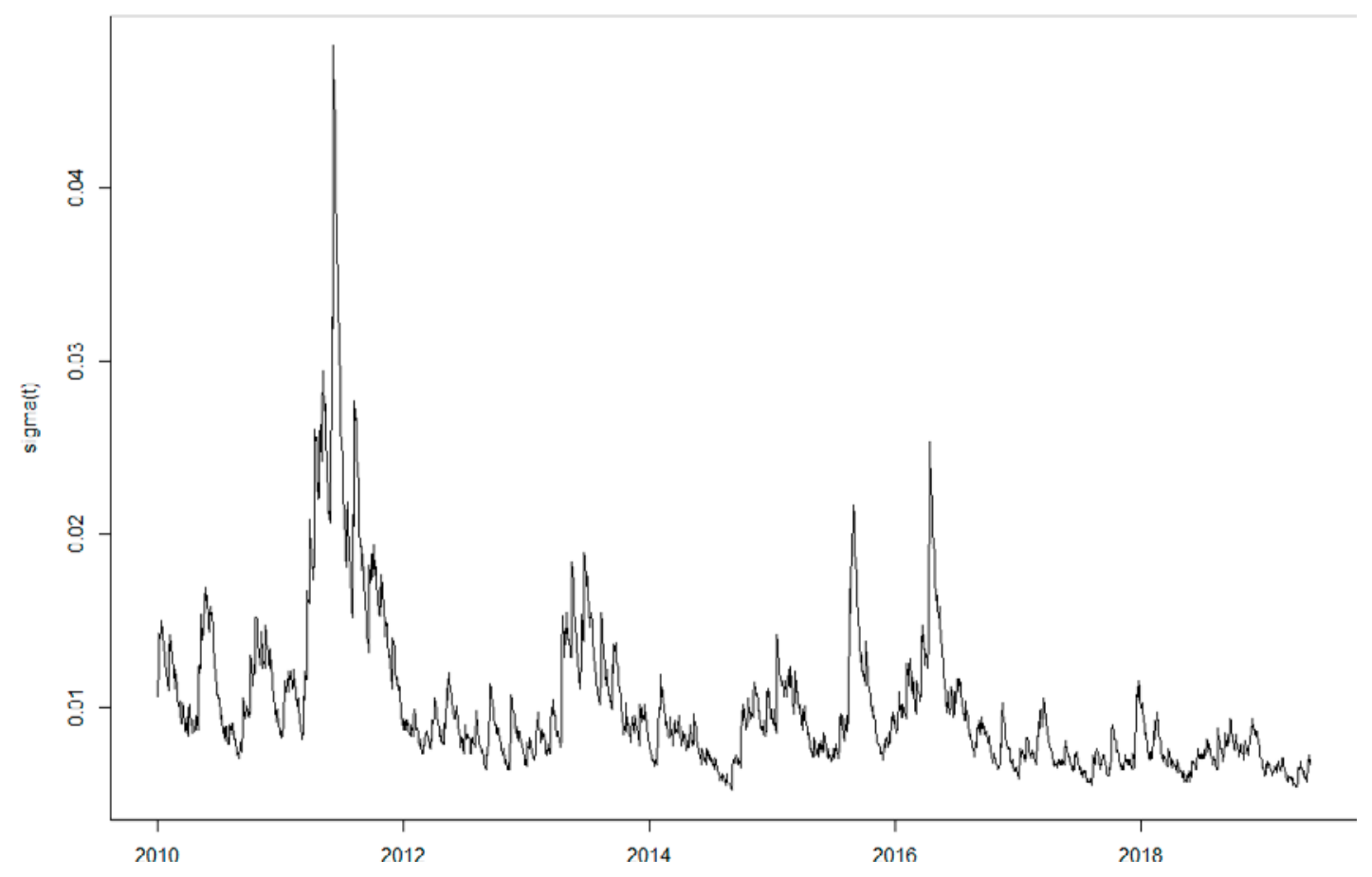

Se puede observar en el gráfico anterior que a grandes rasgos la volatilidad del modelo EWMA y la volatilidad estimada a través del modelo GARCH tienen comportamientos similares.

Considerando una medida alternativa de volatilidad (modelo SV), en la cual se toma en consideración la incertidumbre asociada en la estimación de la varianza, se obtiene una estimación más flexible de la volatilidad del retorno de IGVBL. Así, tomando en consideración la mediana de la distribución de la varianza, obtenemos un estimación de la volatilidad (std) a través de modelo SV que muestra un comportamiento similar a los modelos previos (Gráfica 3). 
Gráfica 3. Medición de volatilidad del IGBVL utilizando el modelo de Volatilidad Estocástica

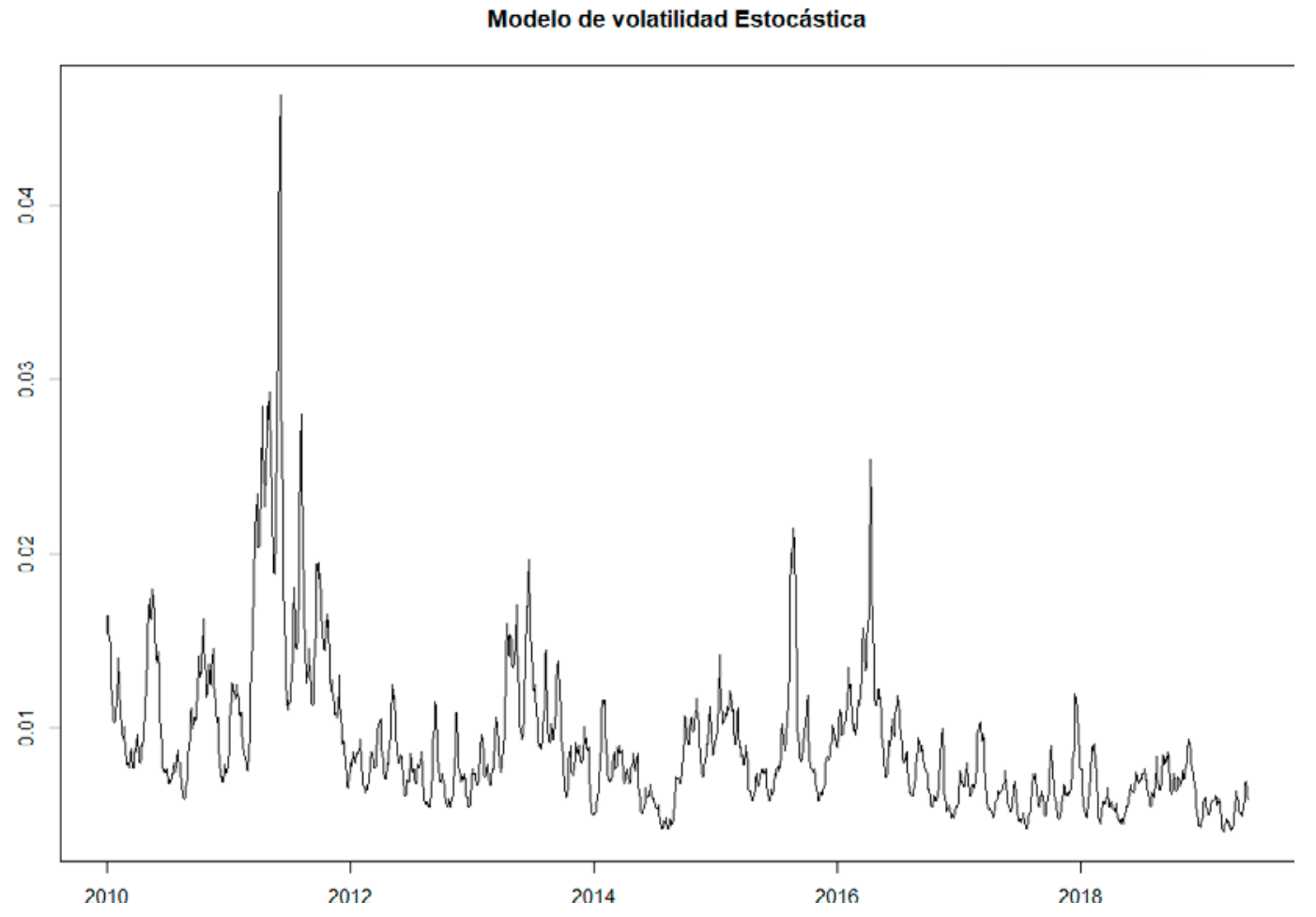

\section{c. Usando el Valor en Riesgo para comparar las medidas de volatilidad}

Para hacer una comparación formal entre las metodologías descritas, se utilizó las volatilidades de cada modelo para el cálculo del Valor en riesgo (VaR) y se procedió a realizar un ejercicio de backtesting para evaluar qué tan efectivo es el Valor en Riesgo de cada uno de los modelos. La estrategia de backtesting consiste en utilizar una muestra móvil de 2000 observaciones con la cual se estima para cada modelo la varianza para el siguiente día y con este resultado se obtiene el Valor en Riesgo para el siguiente día. Este procedimiento se realiza para los restantes 446 días y se obtiene una muestra de igual tamaño para el Valor en Riesgo los cuales se comparan con el valor del retorno bursátil realizado y verificar si dicho retorno excedió el valor en riesgo o no. Una adecuada medida de riesgo debería tener un porcentaje de excepciones en la muestra igual al nivel de significancia. El gráfico 4 muestra tanto el retorno bursátil como las estimaciones del valor en riesgo de las tres metodologías utilizadas (en el eje vertical), donde se pueden identificar los períodos dentro de los 446 días de la muestra donde el retorno bursátil excedió el Valor en Riesgo. 


\section{Gráfica 4. Backtesting Value at Risk (3 modelos)}

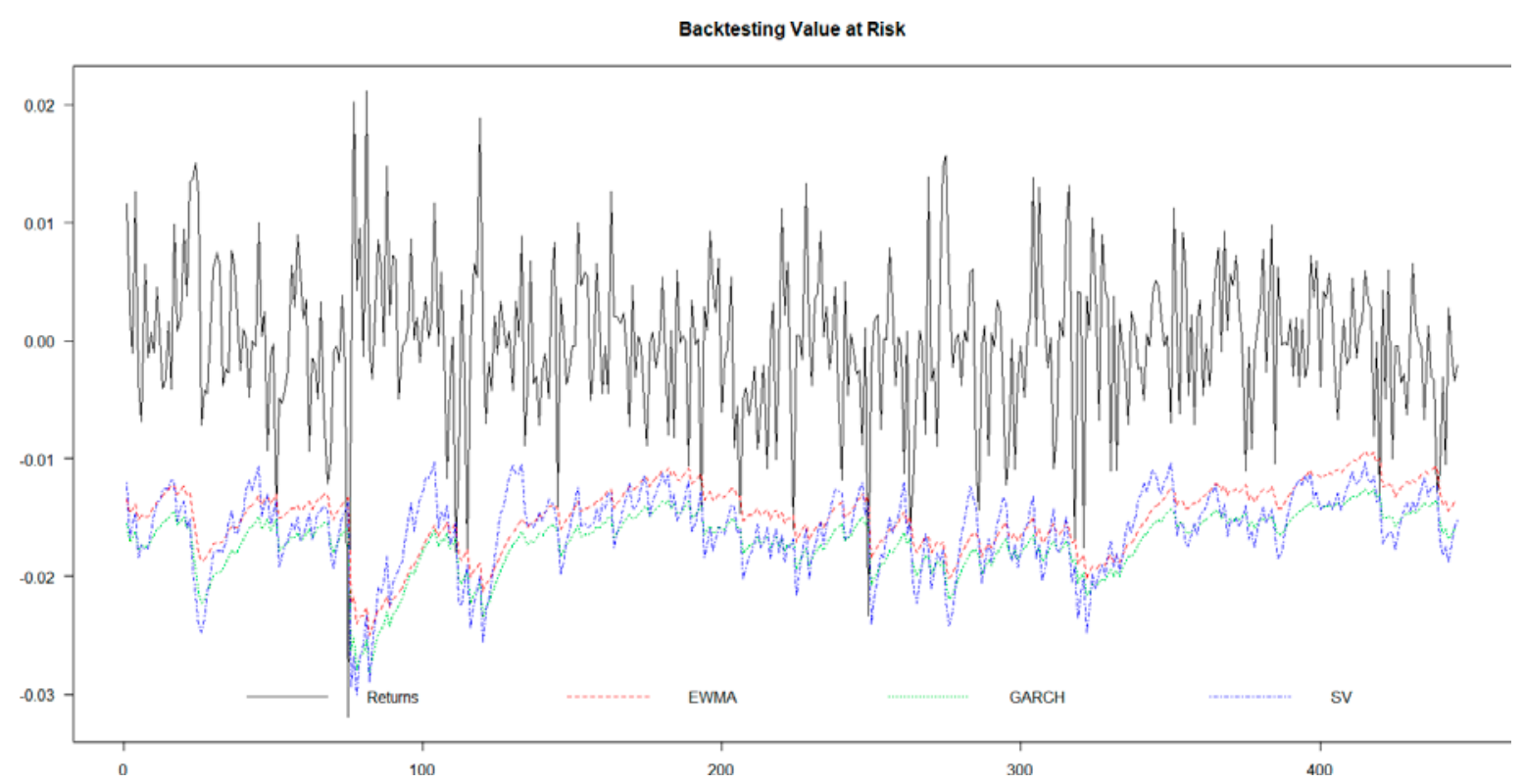

La Tabla 1 muestra que el porcentaje de excepciones que tienen los tres modelos estimados. Se puede observar que tanto el modelo GARCH como el SV tienen un porcentaje de excepciones por debajo del modelo EWMA y consistentes con el nivel de significancia de $1 \%$ utilizado.

Tabla 1. Backtesting VaR para el IGBVL

\begin{tabular}{|c|c|c|}
\hline & $\begin{array}{c}\text { Ratio de excepciones } \\
(\%)\end{array}$ & Volatilidad del VaR \\
\hline EWMA & 2,690583 & 0,002850377 \\
\hline GARCH & 1,121076 & 0,0026265 \\
\hline SV & 1,193722 & 0,003358114 \\
\hline
\end{tabular}

Dado que ex-post VaR no es observable, no se pueden utilizar criterios como ECM de la proyección, por lo que los métodos de backtesting se basan el excepciones. Se define excepción, $I_{\mathrm{t}}$, como:

$$
I_{\mathrm{t}+1}(p)= \begin{cases}1, & X_{t+1}<\chi_{P}^{t+1} \\ 0, & X_{t+1} \geq \chi_{P}^{t+1}\end{cases}
$$

Si el modelo está bien especificado, deberían comprobarse el siguiente principio del backtesting: Hipótesis de cobertura no condicional:

$$
\operatorname{Pr}\left[I_{t}(p)=1\right]=E\left[I_{t}(p)\right]=p
$$

Si el modelo está bien especificado, $I_{t}$ es una secuencia de variables aleatorias con distribución Bernoulli. Prueba de cobertura de Bernoulli (Kupiec, 1995):

$$
H_{0}: I_{t} \sim B(p)
$$

Donde es el nivel de significancia (cobertura) del modelo VaR. Si se considera como, donde $\mathrm{n}$ es el tamaño del período de backtesting, entonces es el estimador muestral de . Bajo la hipótesis nula, tenemos que, lo que podríamos comprobar mediante una prueba de LR:

\section{Tabla 2. Comparación de VaR}

\begin{tabular}{|c|c|c|}
\hline \multirow{2}{*}{ Modelo } & \multicolumn{2}{|c|}{ Cobertura incondicional } \\
\cline { 2 - 3 } & t-statistic & p-value \\
\hline EWMA & 8.804 & 0.003 \\
\hline GARCH & 1.064 & 0.800 \\
\hline SV & 1.297 & 0.530 \\
\hline
\end{tabular}

Comparando la prueba de cobertura condicional, se puede observar que nuevamente el modelo EWMA es inferior a los otros modelos puesto que se rechaza la hipótesis de que el porcentaje de excesos es igual al nivel de significancia. Por su parte, entre los modelos GARCH y SV, en ambos modelos se acepta la hipótesis nula y por lo tanto ambos serian útiles cuando se requiere calcular el Valor en Riesgo. 


\section{Conclusiones}

La volatilidad de los activos financieros es una de las principales medidas requeridas para la gestión de riesgos financieros. Por tal motivo, se necesita una medida confiable de dicha volatilidad. En este artículo se utilizan las principales medidas de varianza para estimar la volatilidad del retorno del índice bursátil de la Bolsa de Valores de Lima. Para hacer comparables estas metodologías se calculó el Valor En riesgo de cada modelo y se realizó un ejercicio de backtesting. Los resultados muestran que si bien las tres metodologías de estimación generan medidas de volatilidad similares, los modelos GARCH y SV son superiores al modelo EWMA en términos del cálculo del Valor en Riesgo. Asimismo, al utilizar el ejercicio de backtesting para comparar la capacidad predictiva de los modelos GARCH y SV, no se encuentras diferencias significativas entre ambos modelos por lo que no se puede concluir que un modelo es superior al otro. Sería de utilidad adicionales pruebas que puedan determinar la superioridad de alguno de estos modelos.

\section{REFERENCIAS}

[1] Bollerslev T (1986). “Generalized Autoregressive Conditional Heteroskedasticity". Journal of Econometrics, 31(3), 307-327.

[2] Bos CS (2012). "Relating Stochastic Volatility Estimation Methods." In L Bauwens, C Hafner, S Laurent (eds.), Handbook of Volatility Models and Their Applications, pp. 147-174. John Wiley \& Sons.

[3] Kastner G (2016). Stochvol: Efficient Bayesian Inference for Stochastic Volatility (SV) Models.

[4] Kim S, Shephard N, Chib S (1998). "Stochastic Volatility: Likelihood Inference and Comparison with ARCH Models." Review of Economic Studies, 65(3), 361393.

[5] Patricia Lengua Lafosse, Gabriel Rodríguez, "An empirical application of a stochastic volatility model with GH skew Student's t-distribution to the volatility of LatinAmerican stock returns", The Quarterly Review of Economics and Finance, Volume 69, 2018, Pages 155-173.
[6] Taylor SJ (1982). "Financial Returns Modelled by the Product of Two Stochastic Processes: A Study of Daily Sugar Prices 1691-79." In OD Anderson (ed.), Time Series Analysis: Theory and Practice 1, pp. 203-226. North-Holland, Amsterdam.

[7] Willy Alanya \& Gabriel Rodríguez, 2014. "Stochastic Volatility in Peruvian Stock Market and Exchange Rate Returns: a Bayesian Approximation," Documentos de Trabajo / Working Papers 2014-392, Departamento de Economía - Pontificia Universidad Católica del Perú.

[8] Kim S, Shephard N, Chib S (1998). "Stochastic Volatility: Likelihood Inference and Comparison with ARCH Models." Review of Economic Studies, 65(3), 361393. 